\title{
Positioning Beacon System Using Digital Camera and LEDs
}

\author{
Hugh Sing Liu, Member, IEEE, and Grantham Pang, Senior Member, IEEE
}

\begin{abstract}
This paper is on a novel use of lighting or signaling devices constructed by light-emitting diodes (LEDs) as a positioning beacon. The idea is that the surface of the LED lighting device is divided into regions and used to show different visual patterns that are not noticeable by the human eye due to the high-frequency switching of the LEDs. A digital camera is used as a receiver to capture a sequence of images of the LED positioning beacon transmitter. Image-processing algorithms are used to decode the location code that is encoded in the visual patterns transmitted by the LED device. This idea can be applied to any LED traffic lights or signaling devices on the road and turn them into parts of a positioning beacon system. Such a system made up of high-brightness visible LEDs can provide the function of open-space wireless broadcasting of the positioning signal. The LED signaling method, transmission protocol, camera frame rate, LED flash rate, together with an implemented system and the experimental results, are presented in this paper.
\end{abstract}

Index Terms-Digital image processing, light-emitting diode (LED), location beacon, vehicle positioning.

\section{INTRODUCTION}

$\mathbf{T}$ HE growth of development in the area of light-emitting diodes (LEDs) is mainly due to the introduction of AlInGaP devices. Nowadays, billions of visible LEDs are produced each year, and the emergence of high-brightness $\mathrm{AlGaAs}$ and AlInGaP devices has given rise to many new markets [1]-[3]. More recently, AlGaInN materials have been introduced and led to improvements in the performance of bluish-green LEDs [1]. The luminous "efficacy peaks" are much higher than incandescent lamps [3], and the entire visible spectrum is not available. This advancement has led to the production of large-area full-color LED displays for outdoors applications.

LEDs are increasingly being used in message display boards, traffic lights, signal devices, or other means of illumination on the road. They are superior to incandescent lights due to their long life expectancy, high tolerance to humidity, low power consumption, and minimal heat generation [4]. One important characteristic of LEDs is that they are semiconductor devices and are capable of switching on and off at a very fast rate. When the LEDs are arranged in a special pattern and switched in a particular way, they could display a special code that is invisible to the human eye. Therefore, in addition to the normal function of being an indication and illumination device, an LED traffic light or signal device can be used as a location-code broadcasting de-

Manuscript received July 2000; revised October 2002.

H. S. Liu is with Saning Electronic Limited (e-mail: hughliu@ieee.org).

G. Pang is with the Department of Electrical and Electronic Engineering, The

University of Hong Kong, Hong Kong, China (e-mail: gpang @eee.hku.hk).

Digital Object Identifier 10.1109/TVT.2002.808800 vice. Hence, it becomes part of a wireless positioning beacon system. This paper describes the architecture and design of such a system for the broadcasting of positioning information. Previous research in beacon vehicle positioning and information system can be found in [5] and [6]

It has been shown that visible light LEDs can be modulated for communication purpose [7]. This concurrent use of visible LEDs for simultaneous signaling and communication leads to many new and interesting applications. The idea of fast switching of LEDs and the modulation of the visible light waves for free space communications has been examined [8]-[12]. The feasibility of such an approach has been demonstrated and hardware has been implemented with experimental results. An optical data link has been implemented using an LED traffic signal head as a transmitter [9], [10]. The LED traffic light can be used for either audio or data transmission. Audio messages can be sent using the LED transmitter, and a receiver located at a distance around $20 \mathrm{~m}$ away can play back the messages with a speaker. For data transmission, digital data can be sent using the same LED transmitter, and experiments have been performed to send a speed-limit information.

The work reported in this paper extends from the previous work in different ways. The view angle of the receiver of the previous work [11], [12] is only around $5^{\circ}$, which is very small. Hence, this gives a direct line-of-sight problem between the transmitter and the receiver. The new development reported in this paper can extend the field of view from $5^{\circ}$ to $30^{\circ}$ in a real application. Another extension is that in the previous work, the LEDs of the traffic light are turned on and off at the same time instance and the receiver is constructed from a photodiode. In this paper, the switching of the LEDs in the transmitter is more sophisticated and a digital camera is used as a receiver. Image-processing algorithms are developed to retrieve the location code from the LED positioning beacon. More about beacon location systems can be found in [13] and [14]

\section{SYSTEM ARCHITECTURE}

The location beacon system developed consists of an intelligent LED traffic light [10] and a camera-based receiver. A location code is transmitted by the intelligent LED traffic light. A passing-by vehicle equipped with the camera-based receiver can obtain this location code through this visible optical wireless link. The scenario is shown in Fig. 1. A block diagram representation of the system is shown in Fig. 2. At the transmitter side, the three major components are the microcontroller, LED driver, and LED panel. The modulated signal is transmitted by the switching of the LEDs. The switching frequency is high enough that the perceivable light appears to be constantly il- 


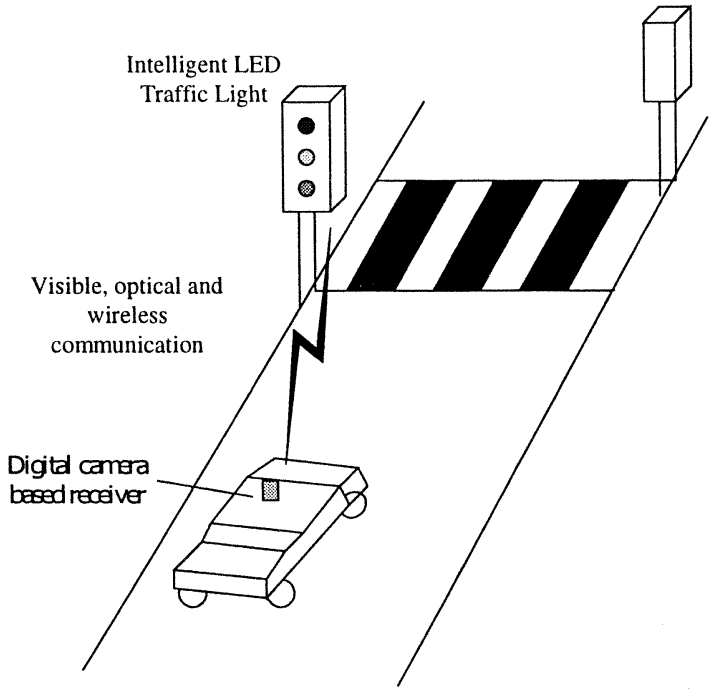

Fig. 1. A scenario of the proposed LED location beacon system based on a digital camera receiver.

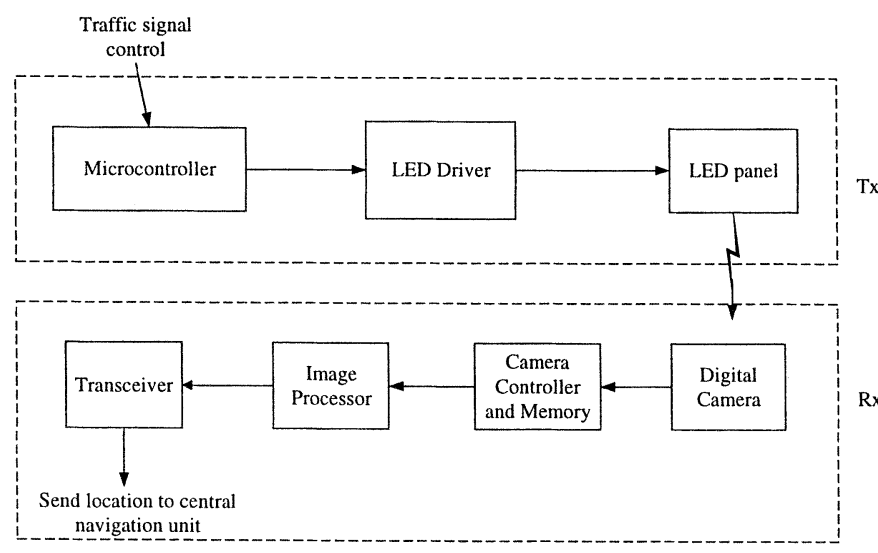

Fig. 2. System architecture of the proposed location beacon system.

luminated to the human eye. The receiver consists of a digital camera, a camera controller, memory units for storing images, an image processor, and a transceiver for external communication.

\section{A. Intelligent LED Traffic Light}

When a photodiode-based receiver is used, all the LEDs in a panel are switched on and off at the same time. When this camera-based receiver is used, the LED panel is partitioned (in this paper, nine partitions are proposed). Within each partition, the LED is turned on and off simultaneously. However, different patterns can be shown on the LED panel at a time. A microcontroller unit (MCU), which contains a unique location code, mainly performs two tasks. First, it is responsible for controlling the on-pattern of the LED panel in different periods. Thus, different patterns on the LED panel are actually being flashed, and the rate is fast enough that the human eye cannot detect it. The sequence of flashes is in accordance with a proposed protocol described later. The second task of the microcontroller is to control the intelligent traffic light so that it functions as a normal traffic signaling device. Driver circuits are necessary to switch the array of LEDs on and off in a fast way and at the same time

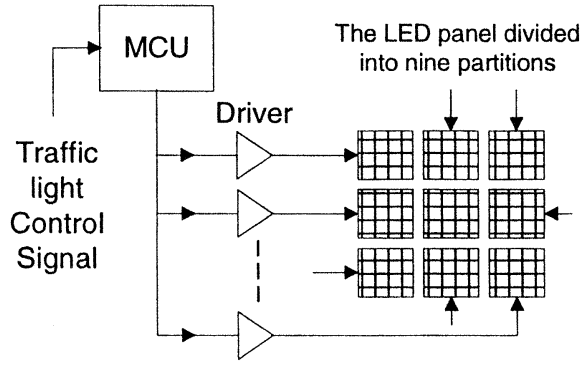

Fig. 3. The architecture of the intelligent LED traffic light for a camera-based receiver.

provides enough current to the LEDs. A power MOSFET can be used in the intelligent LED traffic light design. Fig. 3 shows a diagrammatic illustration of the intelligent LED traffic light.

\section{B. Digital Camera-Based LED Signal Receiver}

The location beacon receiver consists of a digital camera, a camera controller, memories for storing images, an image processor, and a transceiver for external communication. Fig. 4 shows the structure of the digital camera-based LED signal receiver. A CMOS digital camera module is used to capture the images of the intelligent LED traffic light. The operations of the digital camera are controlled by the control unit. The operation of the digital camera is controlled for image capturing and parameter modification. The captured images are stored in the memory unit and then processed. The image processor would extract the location code from the images with the algorithms developed. The transceiver is used for sending out the extracted location code to a main navigation unit. It is a bridge for the communication between the digital camera-based LED signal receiver and the main navigation unit.

\section{TRANSMission PROTOCOL}

The design of the transmission protocol for the intelligent LED traffic light is based on the differencing of image sequences, which can be used to extract the location code. A good transmission protocol would provide a simple and efficient system. The criteria of the protocol design, the proposed A-On-A'-Off protocol, and the method of transmitting more than one digit are described in this section.

There are three requirements on the design of the transmission protocol.

1) The LED panel should transmit information wirelessly and optically.

2) The light emitted by the LED panel should be constant (undetectable flashing).

3) Asynchronous communications.

The captured images are stored in the memory unit for processing. The size of digital image is often large. For a $384 \times 288$ pixel, 8-bit gray-level image, the size is $108 \mathrm{Kbyte}$. Thus the transmission protocol should minimize the number of image frames required for the extraction of a location code. This could save the cost by using less memory and decrease the computational time by processing fewer image frames.

The location beacon is a simplex communication link. The protocol is designed to be simple and workable. Thus, the pro- 


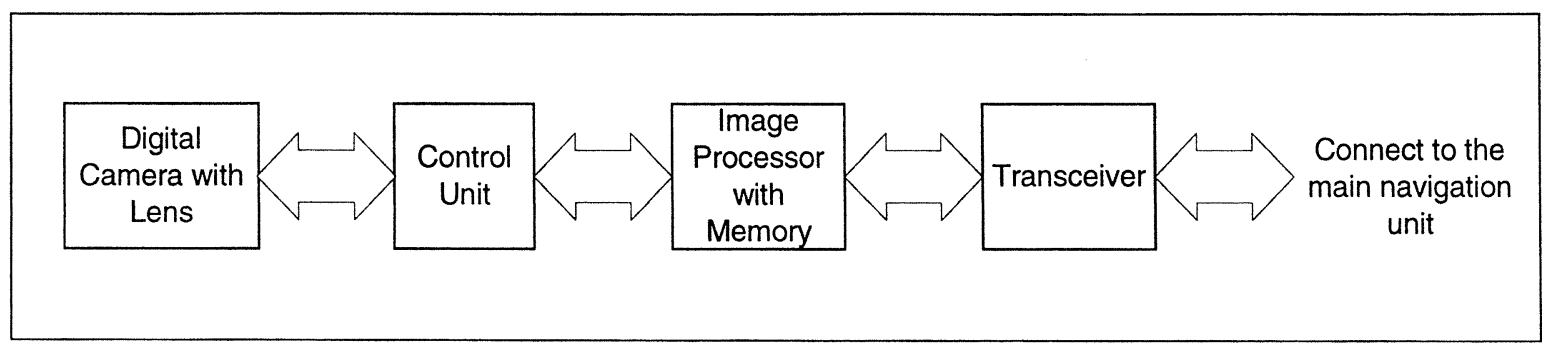

Fig. 4. The structure of the digital camera-based LED signal receiver.

tocol design should prevent the need of synchronization between the transmitter and the receiver. This means that a vehicle can receive a location code at any time it approaches the intelligent LED traffic light.

\section{A. A-On-A'-Off Protocol}

The A-On-A'-Off protocol is proposed to satisfy the requirements stated. Pattern A represents a particular LED pattern as illustrated in Fig. 5, and Pattern A' denotes the complement of A. Pattern On means that all the partitions are turned on and Pattern Off means that all the partitions are turned off.

Using the A-On-A'-Off protocol, the LED patterns A, On, A', and Off are displayed sequentially for a short period. This sequence is repeated periodically. With the fast switching among these patterns, the LED panel appears to be turned on with constant brightness. The reason is due to the perception of the human vision system. When A and A' are flashing at a fast rate alternatively, the LED panel appears to be all on at the same brightness. This phenomenon is also found in the fast switching between On and Off patterns.

The differencing of the LED patterns transmitted in the A-On-A'-Off protocol would have results as shown in Table I.

From Table I, it can be observed that the (absolute of) differencing of neighboring pattern pairs always yields pattern A and A'. If pattern A and A' is assigned to a unique location code, then a differencing process of a received pair of images can yield the location code of an intelligent LED traffic light. Thus, the camera only needs to capture two consecutive images for the differencing process. This minimizes the size of the memory unit. Moreover, no synchronization between the transmitter and the receiver is needed. Any consecutive pairs of patterns can be captured by the camera-based receiver at any time, and a location code can be yielded by performing a differencing process.

One point to note is that if the LED pattern update rate is low, then the flickering effect can be seen. Thus, the minimum LED pattern update rate should be found. An experiment was carried out using a signal generator to give a $50 \%$ duty cycle square wave to drive an LED. It was found that the flashing of the LED is undetectable when the frequency is over $50 \mathrm{~Hz}$. The term $t_{\text {LED_pat }}$ represents the duration of showing a particular LED pattern, and the LED pattern update rate $f_{\text {LED_pat }}$ is given by

$$
f_{\text {LED_pat }}=\frac{1}{t_{\text {LED_pat }}} .
$$

Suppose $f_{\text {LED }}$ is defined to be the flashing rate of the LED panel. Referring to Fig. 5, each partition on the LED panel is

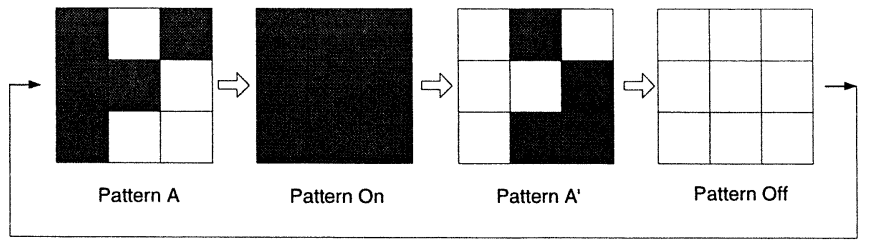

Fig. 5. Pattern transmission sequence using the A-On-A'-Off protocol.

TABLE I

DifFERENCING Results OF NEIGHBORING PATTERn PAIRS TRANSMITTED IN AN A-ON-A'-OFF PROTOCOL

\begin{tabular}{c|c}
\hline $\begin{array}{c}\text { Neighboring Pattern } \\
\text { Pairs }\end{array}$ & $\begin{array}{c}\text { Differencing } \\
\text { Results }\end{array}$ \\
\hline $\mathrm{A}-\mathrm{On}$ & $\mathrm{A}^{\prime}$ \\
\hline On - $\mathrm{A}^{\prime}$ & $\mathrm{A}$ \\
\hline $\mathrm{A}^{\prime}-$ Off & $\mathrm{A}^{\prime}$ \\
\hline Off - A & $\mathrm{A}$ \\
\hline
\end{tabular}

observed to be turned on and off for the duration of $2 * t_{\text {LED_pat }}$ in an A-On-A'-Off cycle. Thus the flashing period that results for each LED partition is $4 * t_{\text {LED_pat }}$ and therefore

$$
f_{\mathrm{LED}}=\frac{1}{4} * t_{\text {LED_pat }} \text {. }
$$

For the LED panel to appear constantly illuminating, the minimum $f_{\text {LED }}$ should be $50 \mathrm{~Hz}$. Hence, the LED pattern update rate $f_{\text {LED_pat }}$, from (1) and (2), should be $4 * 50 \mathrm{~Hz}=200 \mathrm{~Hz}$.

\section{B. Transmitting More Than One Digit}

The intelligent LED traffic light can transmit several digits or even a stream of information to a digital camera-based receiver. After working out all possible combinations and eliminating the overlaps, it was found that an LED panel with nine partitions can present 61 different location codes. Thus a reduced set of printable ASCII character (including A-Z, 0-9, and other symbols) can be represented by an LED pattern. Thus a message can be transmitted if the transmission protocol can handle more than one digit. Also, transmitting more digits can increase the number of unique location codes. In fact, the A-On-A'-Off protocol can be modified in order to support the transmission of several digits. As illustrated in Fig. 6, $\mathrm{H}$ denotes a header pattern. B, C, and D are three different patterns that represent the first, second, and third digit of the location code, respectively. 


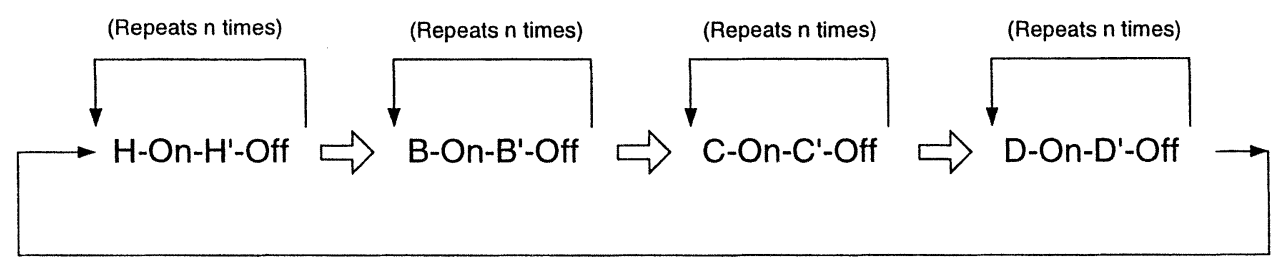

Fig. 6. Example of a transmission protocol sending a three-digit location code.

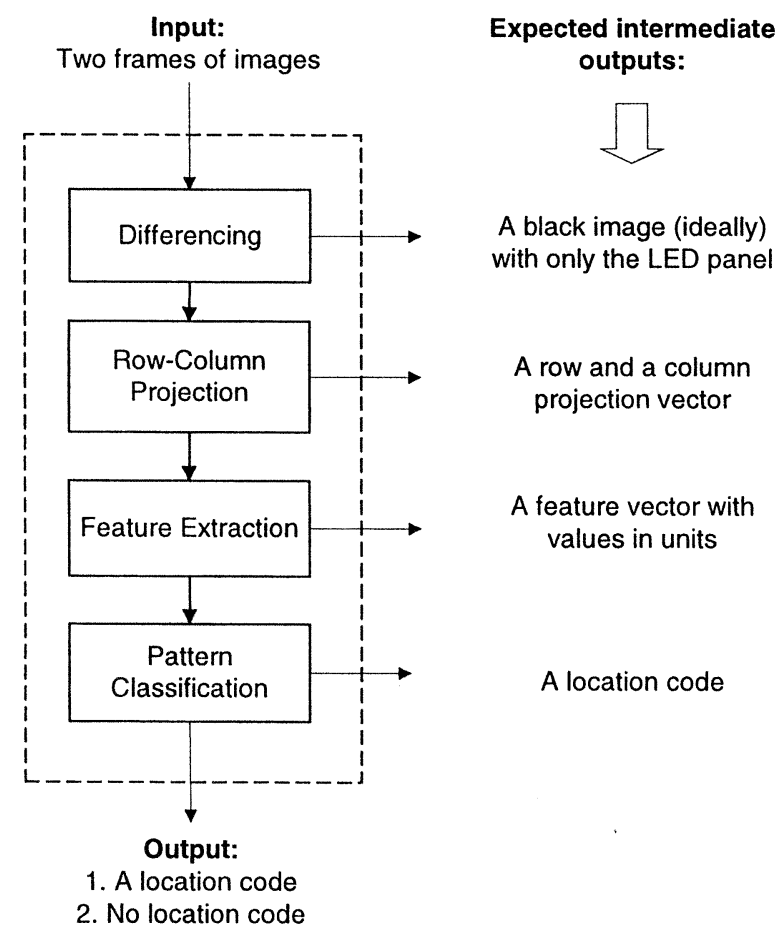

Fig. 7. Flowchart for the recovery of a location code from the captured images.

\section{RECOVERY OF LOCATION CODE FROM THE CAPTURED IMAGES}

After the images are captured by the digital camera and stored in the memory unit, the location data are then extracted. Fig. 7 shows a flowchart for the recovery of a location code from the captured images by using the image-processing algorithms developed.

\section{A. Differencing}

The differencing process is used for detecting whether there is any Intelligent LED Traffic Light signal within the images captured by the digital camera. If there is an LED location beacon signal, this process extracts it from the sequence of images. It works by eliminating the common background within a pair of images and retains only the flashing beacon signal. The differencing process includes the subtraction, absoluting, and noise reduction, which is shown in Fig. 8.

Although the differencing process is a fast and simple method for detecting and extracting the portion of the LED panel within the images, it is affected by the movement of other vehicles in view and its own movement. Two methods are proposed to tackle the problem of noise when differencing due to the movement of objects in the background and the camera movement.

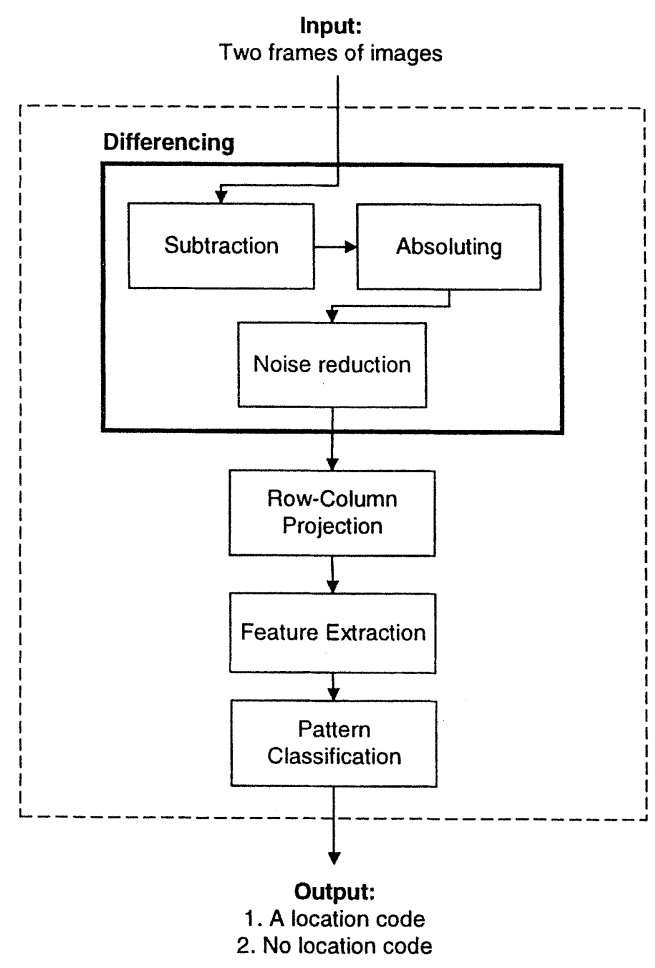

Fig. 8. The differencing processing in the image-processing algorithm.

These methods give a better differenced image for the row and column projection process. The first method is to use a median filter and a thresholding process. The second method involves the use of a red light filter before the camera lens. Moreover, the region of interest of the digital camera can be selected such that the area outside can be excluded. The details of these methods will be described in Section VII.

\section{B. Row and Column Projection}

After the differencing process, the resulting image is used for row and column projection. The purpose of this procedure is to convert the two-dimensional differenced image into a one-dimensional vector to facilitate the feature extraction process. All pixel values in each row are summed and stored in a vector, which is called a row projection vector. The column projection vector is found by summing the pixels in each column. The process in the image-processing algorithm is shown in Fig. 9.

An example of the row and column projection process is shown in Fig. 10. Let $h$ be an integer value showing the height of a projection peak. The resulting row projection vector contains the integer value corresponding to the height of the differenced image, which is [2 12 2] in Fig. 12. Similarly, the column projection vector is [2 3]. 


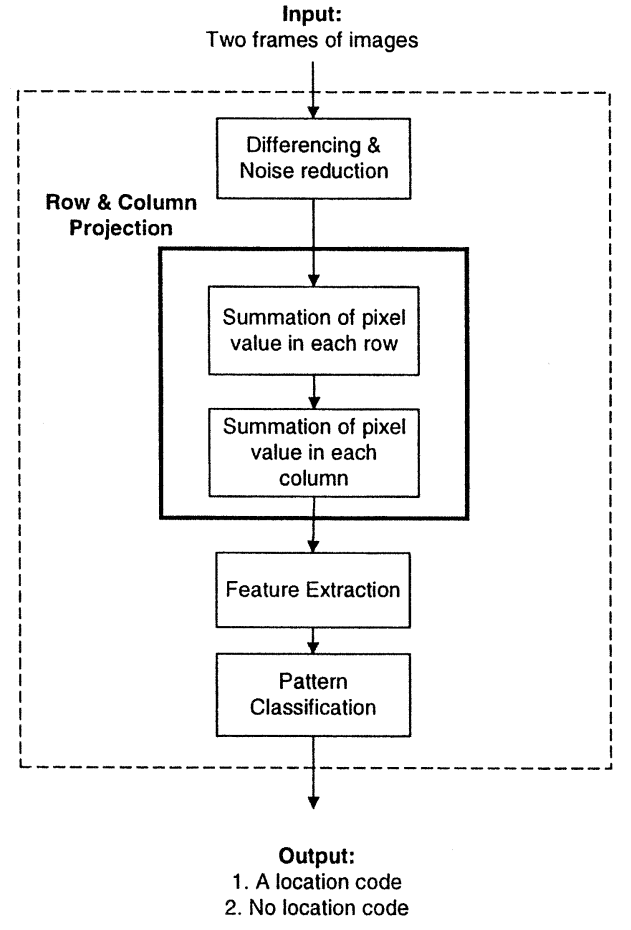

Fig. 9. The row and column projection processing in the image-processing algorithm.

\section{Feature Extraction}

The row and column projection vectors are cascaded to obtain the feature vector for the pattern. The feature extraction processing in the image process algorithms is shown in Fig. 11. For example, the feature vector for the pattern in Fig. 12 should be

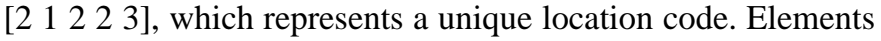
from the top down in the row projection and then left to right in the column projection are processed and give the feature vector. In an actual situation, the row and column projections waveform may not be so precise due to noise and distortion of the LED panel signal. Thus, a robust algorithm for finding the peaks and the corresponding values in the differenced image is needed in order to provide correct results.

1) Feature Vectors for the $3 \times 3$ LED Panel: For a $3 \times 3$ LED panel, there should be $2^{9}$ or 512 different patterns. However, not all of them can be used for representing a location code. This is due to the nature of the row and column projection and the feature extraction process. The reason is illustrated in Fig. 12. In Fig. 12(a), the horizontal projection gives values of $\left[\begin{array}{lll}2 & 1 & 0\end{array}\right]$ for the three rows. For vertical projection, the three values are [l $\left.\begin{array}{lll}2 & 1 & 0\end{array}\right]$. The value of zero (i.e., [0]) is then ignored. When the horizontal projection value is cascaded with the vertical projection value, we obtain the feature vector [ $\left.\begin{array}{llll}2 & 1 & 2 & 1\end{array}\right]$. In Fig. 12(b), horizontal projection gives $\left[\begin{array}{lll}0 & 2 & 1\end{array}\right]$ and vertical projection gives [ $\left[\begin{array}{lll}2 & 1 & 0\end{array}\right]$, but the final feature vector is also [ [ $\left.\begin{array}{llll}2 & 1 & 2 & 1\end{array}\right]$. Similarly, all four different LED patterns have the same feature vector [ [ $\left.\begin{array}{llll}2 & 1 & 2 & 1\end{array}\right]$ and can represent just one location code. Thus, the number of usable patterns is reduced. Moreover, a pattern and its complement pattern are both assigned the same unique location code due to the design of the transmission protocol. This further reduces the number of location codes that can be presented by the LED panel. Finally, only 61 pairs of the LED patterns with their complements can be used to present the location codes. As an example, nine of the LED patterns and their complements are shown in Fig. 13. These nine pairs of feature vectors represent nine LED pattern-complement pairs (referring to Fig. 13), as shown in Table II.

The number of location codes that can be presented by the LED panel can be increased by sending a multidigit code, as mentioned earlier. However, the multidigit code cannot contain an identical neighboring digit due to the transmission protocol design. Also, there should be a digit reserved for the header. Thus, for a three-digit location code, the number of location codes supported should be $60 \times 59 \times 59=208860$.

\section{Pattern Classification}

Once the feature vector is obtained, the corresponding location code can be found by searching a lookup table relating the feature vectors and the location codes. For a fast search, the feature vector data can be organized into a tree structure with three branches (for values of one, two, and three in the feature vector). For a $3 \times 3$ LED panel, the feature vector for a location code digit contains zero to six elements. Thus, the tree structure contains six levels. A searching algorithm such as heuristic search and bidirectional search can be used.

\section{NOISE IN THE DIFFERENCING}

Although the differencing process is a fast and simple method for detecting and extracting the portion of the LED panel within the images, it is affected by the movement of other vehicles in view and its own movement. The concept of differencing given before is based on the assumption of a static background except the signal and a static camera. In this section, an analysis of noise caused by the object and the camera movement is given.

\section{A. Object Movement}

The objects in the background could have moved between two consecutive image frames. Thus, the result of the differencing contains the signal as well as the noise caused by the background objects that may have shifted slightly. An analysis is to be developed for the study of how the system parameters are related to the noise.

The following terms are defined:

$F \quad$ camera capture rate in frame per second (fps);

$v \quad$ object speed $\left(\mathrm{ms}^{-1}\right)$;

$D$ perpendicular distance from the camera lens to the object (m);

pixel width of the digital camera $(\mathrm{m})$;

focal length of the camera lens $(\mathrm{mm})$;

distance between the lens and the camera sensor plane $(\mathrm{m})$;

$M \quad$ magnification factor of the lens;

$x \quad$ displacement of the moving object between two consecutive image frames $(\mathrm{m})$;

$y \quad$ displacement of the image of the moving object on the sensor plane between two consecutive image frames (m); 


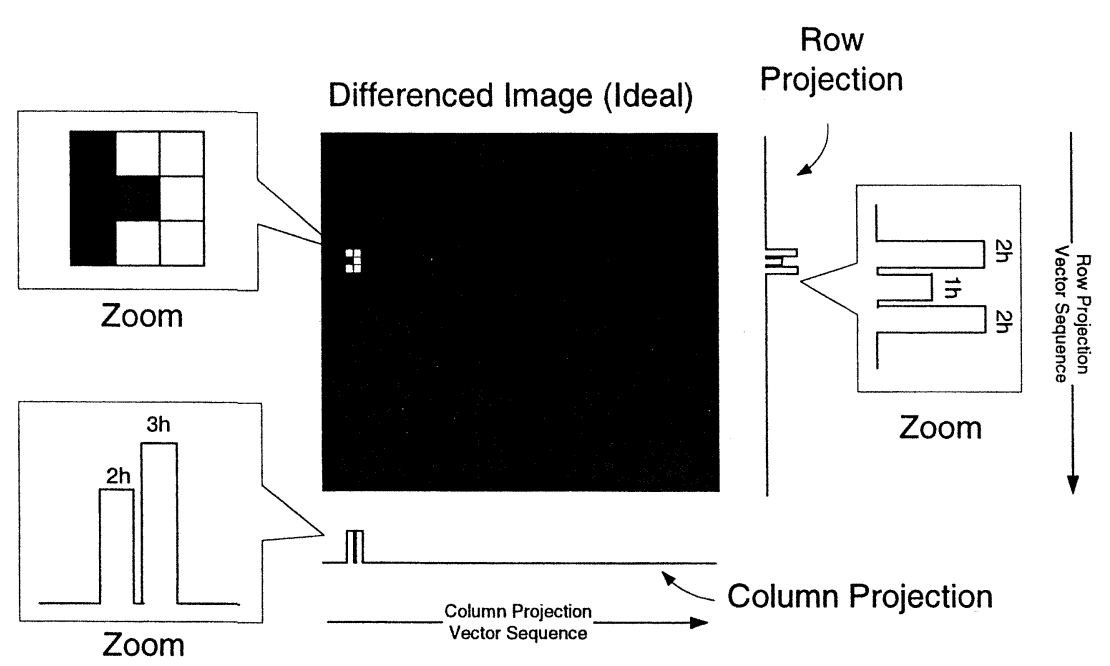

Fig. 10. An example of the row and column projection process.
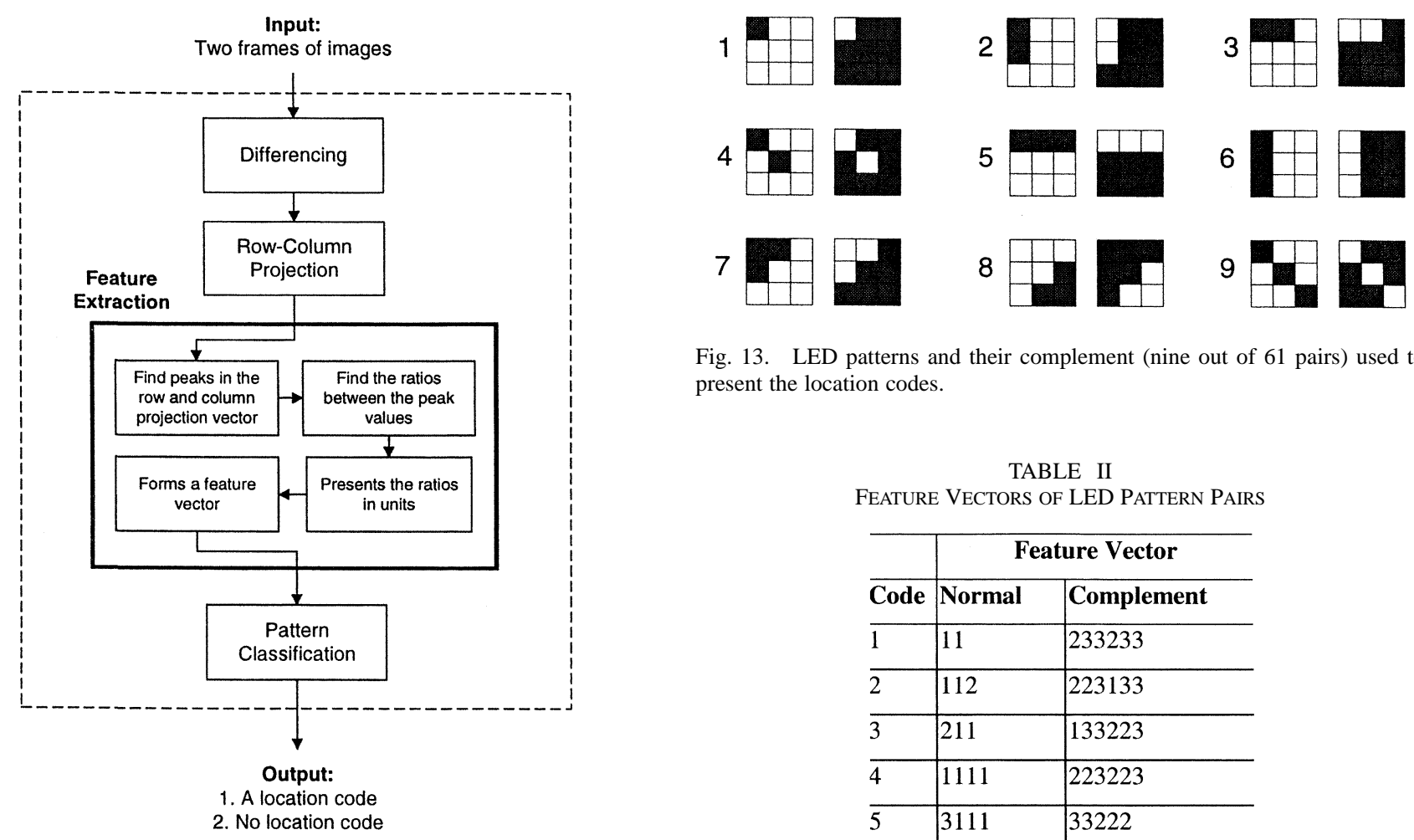

Fig. 13. LED patterns and their complement (nine out of 61 pairs) used to present the location codes.

TABLE II

FEATURE VeCTORS OF LED PATTERN PAIRS

\begin{tabular}{l|l|l}
\hline & \multicolumn{2}{|c}{ Feature Vector } \\
\hline Code & Normal & Complement \\
\hline 1 & 11 & 233233 \\
\hline 2 & 112 & 223133 \\
\hline 3 & 211 & 133223 \\
\hline 4 & 1111 & 223223 \\
\hline 5 & 3111 & 33222 \\
\hline 6 & 1113 & 22233 \\
\hline 7 & 2121 & 123123 \\
\hline 8 & 1212 & 321321 \\
\hline 9 & 111111 & 222222 \\
\hline
\end{tabular}

Fig. 11. The feature extraction processing in the image process algorithms.

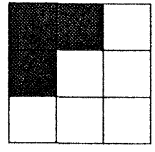

(a)

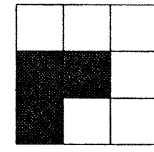

(b)

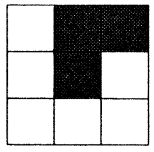

(c)

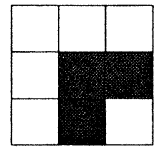

(d)

Fig. 12. Four different LED patterns having the same feature vector.

pix

displacement of the image of the moving object denoted by the number of pixel on the sensor plane between two consecutive image frames.

The case when an object is moving laterally in front of the camera system is studied and illustrated in Fig. 14.

Row

By the principle of optics

$$
M=\frac{y}{x}=\frac{l}{D} .
$$

Then

$$
y=\frac{l}{D} x .
$$




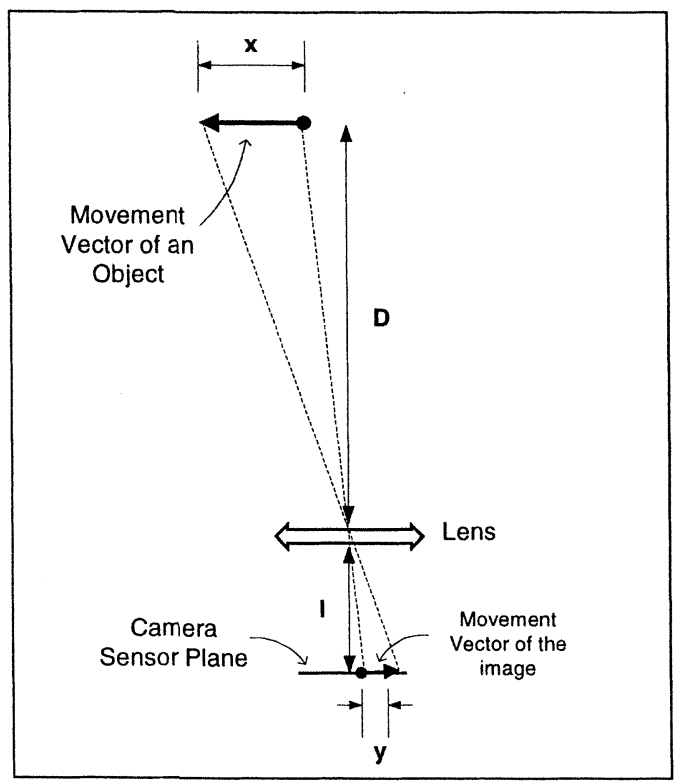

Fig. 14. Illustration of the case when an object is moving laterally in front of the camera system

The displacement of the object between two image frames is

$$
x=\frac{v}{F} .
$$

The displacement of the image of the object on the sensor plane is found by combining (4) and (5)

$$
y=\frac{v l}{F D} .
$$

Hence, the displacement of the image on the sensor plate in terms of the number of pixels is

$$
p i x_{o b j e c t}=\frac{v l}{F D p} .
$$

A numerical example is given below. Assume that a digital camera is kept stationary and takes two pictures at a capturing rate of 50 frames per second. A vehicle is moving laterally at a speed of $45 \mathrm{~km} / \mathrm{h}$ or $12.5 \mathrm{~ms}^{-1}$ (the speed limit is $50 \mathrm{~km} / \mathrm{h}$ for the vast majority of urban roads [15]) at a distance of $15 \mathrm{~m}$ from the camera. The distance between the camera lens and the CMOS sensor plane is $0.02 \mathrm{~m}$. The camera pixel width is 11 $\mu \mathrm{m}$. Then, the change in the distance of the object between the first and the second frame (in number of pixels) is

$$
\text { pix }_{\text {object }}=\frac{12.5 \times 0.02}{50 \times 15 \times 11 \times 10^{-6}}=30.3 \text {. }
$$

This value is too large for the differencing process to be useful. However, if the camera capturing rate is $500 \mathrm{fps}$, then the resulting deviation, which could be only three pixels, is usable for the location code extraction.

\section{B. Camera Movement}

If the camera has been moved laterally relative to an object, then the analysis is similar to the previous one. But suppose the camera is moving toward a point object. Fig. 16 shows an illustration for this case. Let $a$ be the perpendicular distance from the point object to the principal axis of the lens in meters. Let $b$ be the perpendicular distance between the point image to the principal axis of the lens in meters. As the camera moves forward, $b$ would increase.

By the principle of optics, assuming that $l$ is changed according to $D$ in an autofocusing system

$$
\begin{aligned}
M & =\frac{b}{a}=\frac{l}{D} \\
\frac{1}{f} & =\frac{1}{l}+\frac{1}{D} .
\end{aligned}
$$

Suppose that the effect of $\Delta D$ on $\Delta b$ is of interest. By differentiating (8)

$$
\frac{d b}{d D}=a \frac{d\left(\frac{l}{D}\right)}{d D}
$$

by (9)

$$
\frac{l}{D}=\frac{f}{D-f} .
$$

Differentiating this equation gives

$$
\frac{d\left(\frac{l}{D}\right)}{d D}=-\frac{f}{(D-f)^{2}} .
$$

Combining (10) and (11)

$$
\frac{d b}{d D}=-\frac{a f}{(D-f)^{2}} .
$$

Thus

$$
\Delta b=-\frac{a f}{(D-f)^{2}} \Delta D
$$

but $\Delta D=v / F$. Then

$$
\Delta b=-\frac{a f v}{F(D-f)^{2}}
$$

and the change of position of an image (in number of pixels) is

$$
\operatorname{pix}_{\text {camera }}=-\frac{a f v}{F p(D-f)^{2}} .
$$

Assume that the digital camera is mounted on a vehicle moving at a speed of $45 \mathrm{~km} / \mathrm{h}$ or $12.5 \mathrm{~ms}^{-1}$ toward a point object. The point object is at a perpendicular distance of $5 \mathrm{~m}$ to the principal axis from the lens. Two pictures are captured at a rate of $50 \mathrm{fps}$. The object is stationary and initially at a perpendicular distance of $15 \mathrm{~m}$ from the camera. The focal length of the camera lens is $7.4 \mathrm{~mm}$. The camera pixel width is $11 \mu \mathrm{m}$. Then the distance change of the object between the first and the second frame (in number of pixels) is, by (13)

$$
p i x_{\text {camera }}=-\frac{5 \times 0.0074 \times 12.5}{50 \times 11 \times 10^{-6} \times(15-0.0074)^{2}}=-3.74 \text {. }
$$

Thus, from this example, the effect of the vehicle movement toward an object is less severe than the lateral object movement described in the previous subsection. 


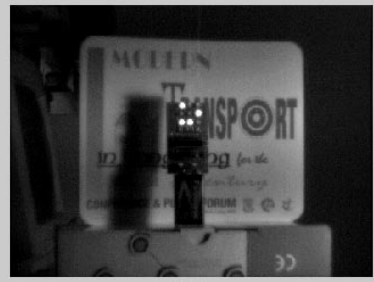

(1)

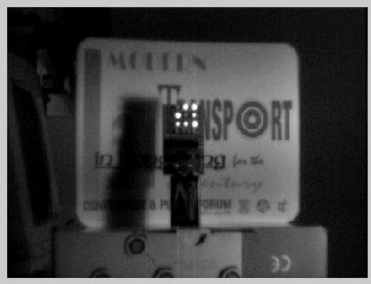

(3)

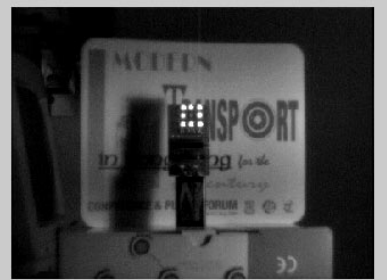

(2)

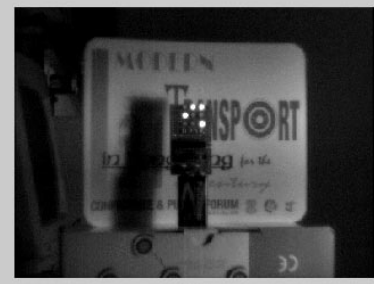

(4)

Fig. 15. Four images captured under a dimmer background and with smaller LEDs.

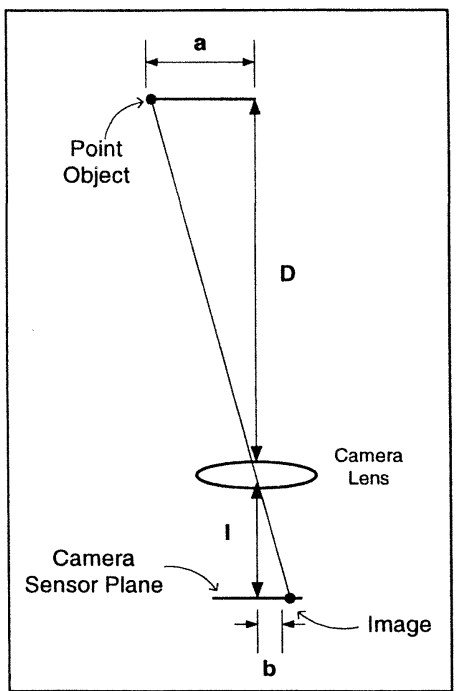

Fig. 16. Illustration of the case when the camera is moving toward an object.

\section{Reduction of Noise in the Differencing}

Here, the problem of noise due to differencing is tackled. The median filter is used to remove the isolated noise. The size of the noise that can be removed depends on the radius of the filter. The thresholding method converts the differenced image, which is in grayscale or color, to a binary black and white image according to a thresholding value. The noise due to the movements of the camera and the background objects may be reduced using these processings.

Another method to reduce the noise in differencing is to use a red filter in front of the camera lens. It has been found effective in reducing noise due to a lateral deviation between two images for as many as ten pixels. With the red filter, the whole image captured by the digital camera is in red but with different intensities. The red light signal of the intelligent LED traffic light has larger intensity in the red image. However, other surrounding items usually have lower intensities. After differencing, almost only the LED panel remains.

Another idea to remove the background noise outside the LED signal area is to use a random pixel access CMOS camera. Once the location of the LED signal within an image is found, the camera only need capture the image on the region of interest (which can be at a very fast frame rate of more than $1000 \mathrm{fps}$ ). Thus, the captured image mostly contains the LED signal only.

\section{ApPlications of the Positioning BeACON System}

We shall now discuss potential applications of the above positioning system. One application is for LED traffic lights to transmit location code information. Nowadays, high-brightness LEDs are increasingly being used in traffic lights. This is mainly due to the low power consumption and minimal maintenance required for LED-based traffic lights, which can be translated into considerable cost savings each year [4], [16]. For example, in Philadelphia, PA, the city is replacing all of its 28000 red signals with LEDs, with an estimated annual cost savings of $\$ 1.2$ million. The next stage of development will involve the entire LED traffic light. With the idea and development described in this paper, an LED traffic light can be used as a positioning beacon, in addition to its normal function of being an indication and signaling device. A receiver some distance away pointing at the traffic light can receive the location code as an alternative to the GPS system. This is especially useful in highly urbanized cities where the urban canyons fail to receive GPS signals due to the blocking of satellites by tall buildings. The above development allows a concurrent use of traffic lights because it can broadcast the location information to both pedestrians and road users and simultaneously perform its normal function of being a traffic-signaling device. The LED traffic light becomes a new kind of short-range information beacon. Essentially, all LED-based traffic signs, displays, or illumination devices can perform the above functions. 


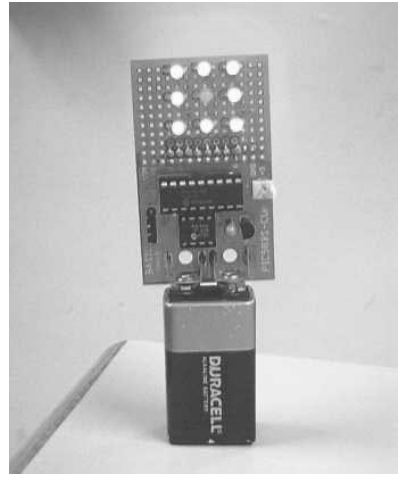

Fig. 17. The eight-LED transmitter prototype (constantly illuminating and transmitting information).

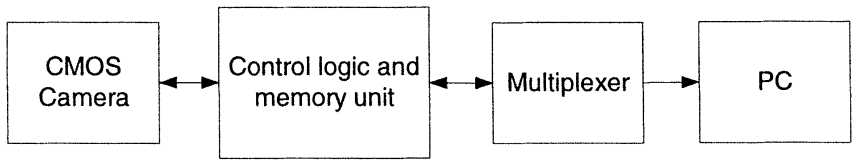

Fig. 18. Block diagram of the digital camera receiver prototype.

\section{EXPERIMENTS ON OBTAINING A LOCATION CODE BY PROCESSING OF LED IMAGES}

\section{A. Transmitter and Receiver Prototypes}

1) LED Transmitter Prototype: A prototype LED transmitter panel consisting of eight LEDs (the center LED is not used) is used to transmit data. LED patterns are updated on the panel in a fast way such that a constant illumination is seen by the human eye. By flashing the LED patterns in the A-On-A'-Off protocol, a location code is transmitted by the LED panel. The transmitter prototype consists of a microcontroller (a BASIC stamp) and the eight LEDs. The A-On-A'-Off protocol is programmed in the EEPROM of the microcontroller. It commands the LEDs to transmit the patterns with a pattern update rate of $250 \mathrm{~Hz}$. Under this frequency, the LED panel is seen to be constantly illuminating. Two sizes of LEDs are used for evaluating the effect of spacing between the LED partitions. Each LED represents a partition in the intelligent LED traffic light. Fig. 17 shows the LED transmitter developed for the experiments.

2) Digital Camera Receiver Prototype: The digital camera receiver can capture and store four frames of $384 \times 288$ grayscale image from the digital camera each time. The four images stored in the memory unit can be transferred to a PC through the printer port. A PC is used to control the camera receiver prototype. It can start the image capturing action, and modify and monitor the parameters of the digital camera such as the exposure time and the sensitivity. The prototype receiver consists of a digital camera, a control logic unit, a memory unit, a multiplexer, and a PC. The structure of the camera receiver is shown in Fig. 18. The OmniVision OV5017 CMOS imager [17] is used in this experiment set. The photographs of the camera module with and without a lens are shown in Fig. 19.

3) Experimental Procedures: The LED panel was set to flash at the patterns as shown in Fig. 20 using the A-On-A'-Off protocol. The LED panel has eight LEDs, which represent eight partitions in the intelligent LED traffic light. The LED panel is

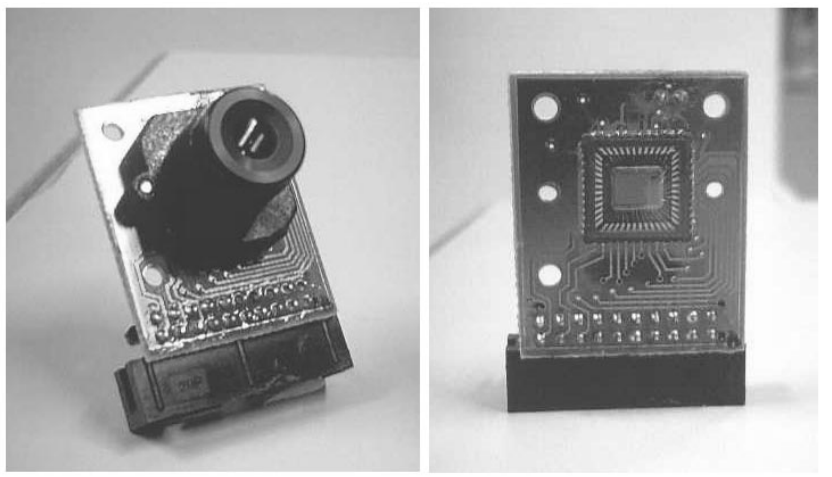

Fig. 19. The OmniVision CMOS camera module (with and without a lens) employed in the beacon receiver.

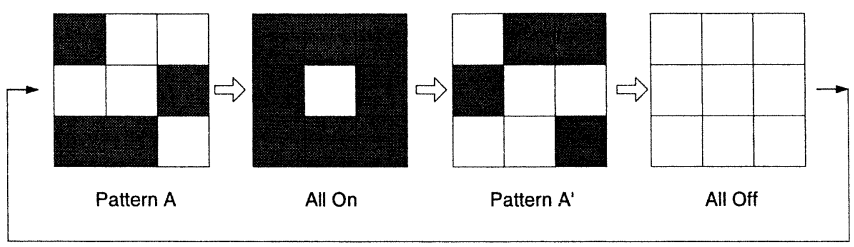

Fig. 20. The LED patterns transmitted with the A-On-A'-Off protocol in this set of experiments.

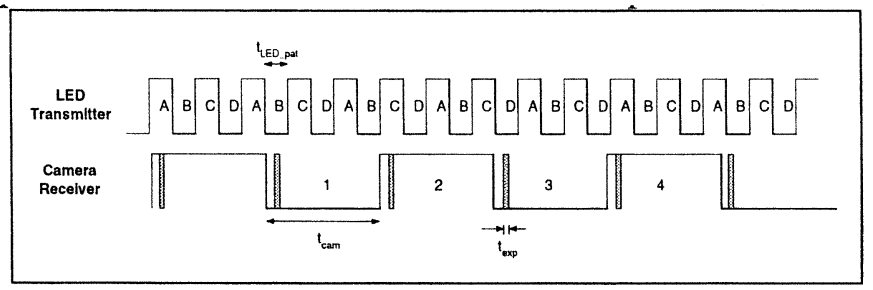

Fig. 21. The timing relationship between the LED transmitter and the camera receiver.

constantly illuminating while transmitting the information. The digital camera receiver is connected to a PC, which controls the operation and get image data from it. Four frames of 8-bit grayscale image with size $384 \times 288$ pixels are captured into the PC from the digital camera. The capturing rate of the digital camera is $50 \mathrm{fps}$ and the shuttering technology employed is electronic rolling shutter. It must be mentioned that the exposure time of a digital camera is different from the frame rate. To avoid the need to use a fast frame rate camera in the experiment, we have used a method as shown in Fig. 21. In the experiment, the LED pattern update rate was $250 \mathrm{~Hz}$. Hence, $t_{\text {LED_PAT }}$ is $1 / 250$ and $t_{\mathrm{CAM}}$ is $1 / 50$. The exposure times of the camera receiver are shown by the shaded regions. This method will ensure that the camera is capturing consecutive pattern update of the LED transmitter. The transitional pattern is not captured for data decoding. Both the receiver and the transmitter are kept stationary during the experiment. The four captured images are processed with the methods described before. The image-processing algorithms are implemented in MATLAB 5.3.

4) Experiment: Dimmer Background With Smaller LEDs: The four captured images with a dimmer background light are shown in Fig. 15. Frame 3 in Fig. 15 is a transitional pattern. Others are good images, which is useful 


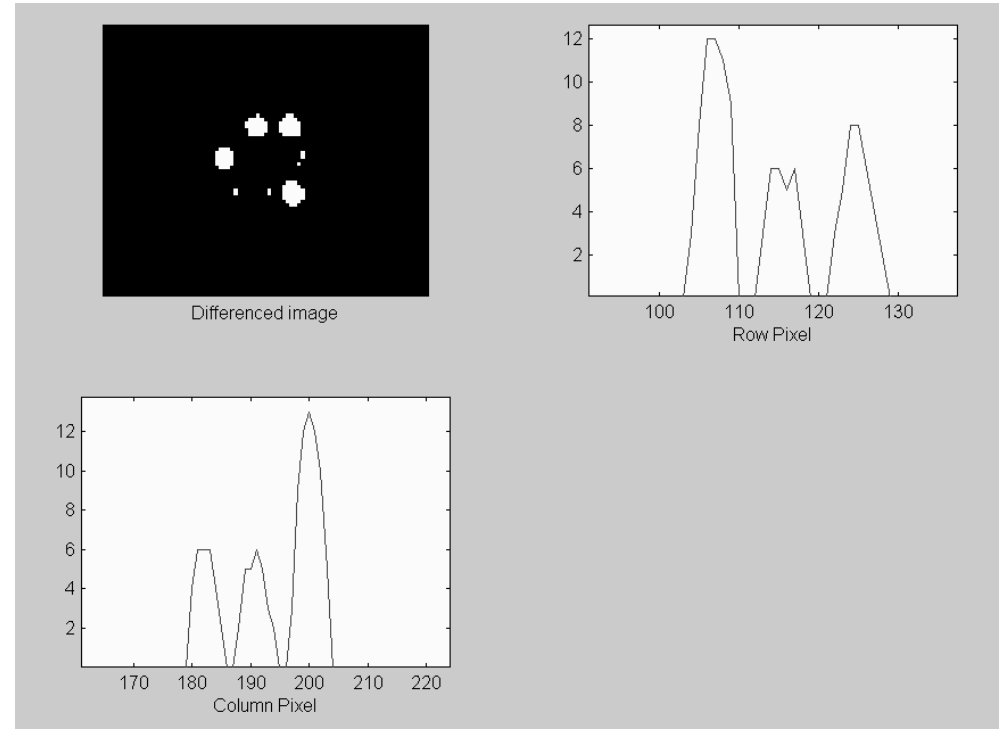

Fig. 22. Closeup of the differenced image of frames 1 and 2 with the row and column projections.

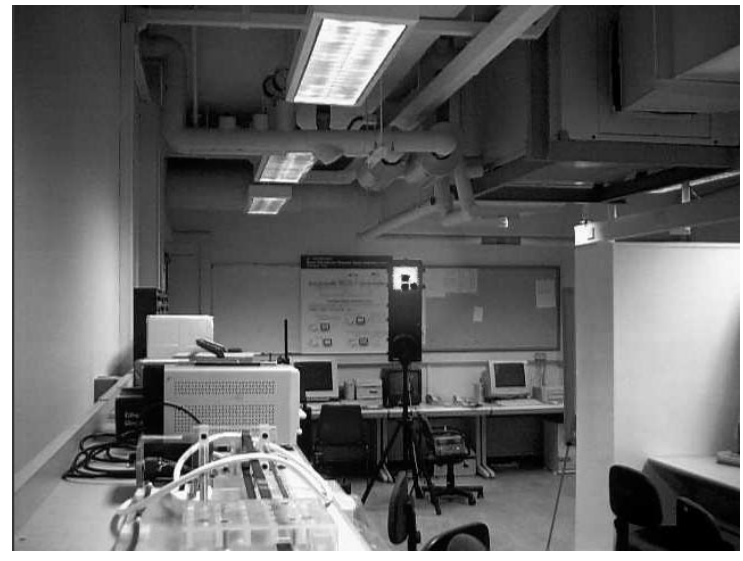

Fig. 23. Image with a LED Traffic light showing a pattern A.

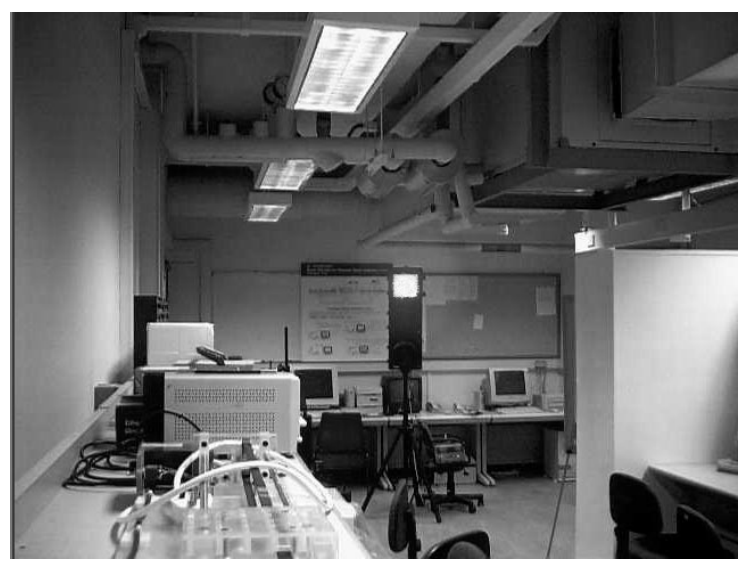

Fig. 24. Image with the LED traffic light turned all on and shifted 2 pixels to the right.

for finding the location code. Fig. 22 shows the zoomed differenced images for patterns (1), (2) and the corresponding row and column projection curves. A thresholding with index 0.3 (ranges from zero to one) is used to convert the grayscale differenced image into a black and white image before the row

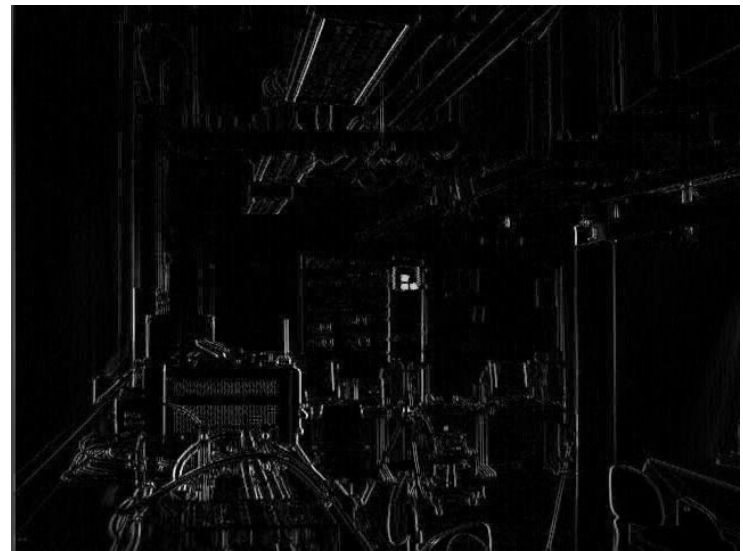

Fig. 25. A differenced image of Figs. 23 and 24 showing the complement (A') of pattern A with noises.

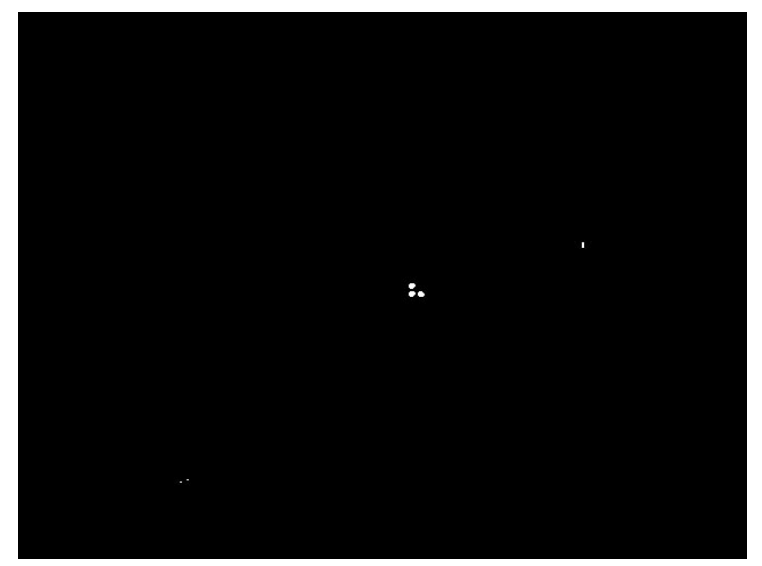

Fig. 26. Image after applying a thresholding process of level 160 (of 256) and three times the median filtering with radius of one pixel to Fig. 25.

and column projection procedure. The location code can be retrieved from the feature vector [ $\left[\begin{array}{llllll}2 & 1 & 1 & 1 & 1 & 2\end{array}\right]$. The computation time required for the four stages of processing on a pair of images is $0.11 \mathrm{~s}$. The result is obtained from running MATLAB 


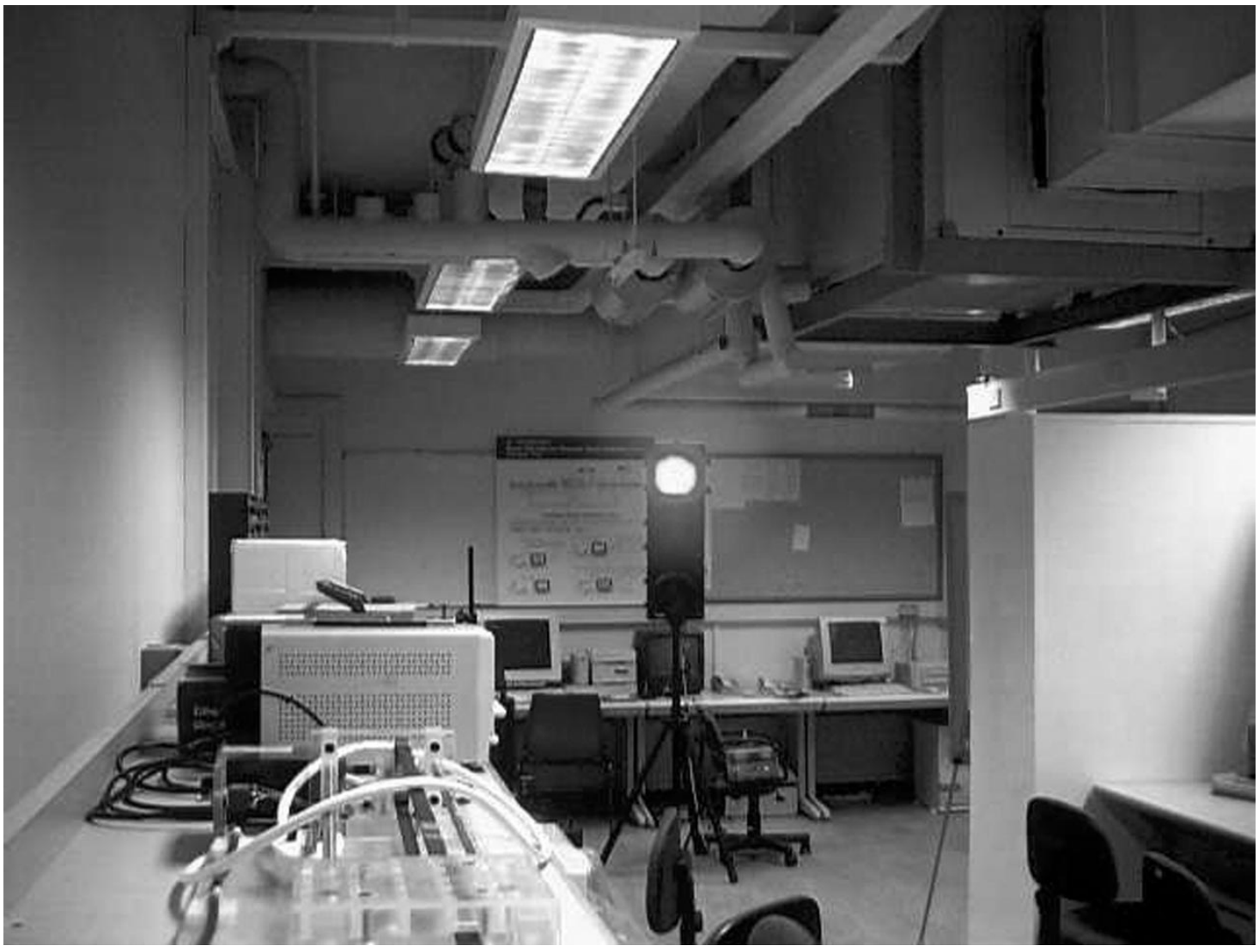

Fig. 27. LED traffic light with the LED turned on.

5.3 on a Pentium III $600-\mathrm{MHz}$ computer. The image size is $384 \times 288$ pixels.

\section{B. Discussion of the Experimental Result}

The proposed location beacon system based on image processing of the LED images has been demonstrated in the above experiments. The location code can be retrieved from the four LED images captured successfully. These verify the ideas and designs of the proposed location beacon. Also, experimental results have shown that even under low signal-to-noise ratio (bright background), the system can retrieve the location code by using the image-processing algorithms.

Although transitional patterns are captured, the program can handle this situation. Also, the chance of getting a transitional pattern is reduced by keeping the proportion of the LED transmitter small in the whole image (larger separation between the transmitter and the receiver). This is to keep the line by line exposure time delay small within the LED portion. The row and column projections are not as precise as the theoretical case. Thus, the feature extraction process is designed with the ability to handle the nonsymmetry and noise of the projection peak portions.

\section{Experiments on Reducing Noise in the Differencing Process}

The purpose of these experiments is to reduce noise in the differenced image due to the background object movements and the camera movement between the time of capturing two frames of image. The thresholding and median filtering process are employed. The results are shown to be dependent on the size of the LED signal in the image and the extent of the noise due to the object and camera movements.

1) Median Filtering and Thresholding: The reduction of noise in differencing by the median filtering and thresholding method is studied. The median filter is useful for removing isolated noises. The size of the noise to be removed depends on the radius of the filter. However, median filtering may also filter out the LED signal partitions. Thus, only the median filter with a small radius can be used for removing limited amounts of noise. The thresholding process changes a graylevel image into a black and white one according to a thresholding index. This helps to remove the noise with intensity smaller than the LED signal.

Fig. 23 shows an image containing a traffic light with a simulated pattern A. Fig. 24 is an image similar to Fig. 23, but with the LED pattern all-on and the image shifted to the right by two pixels. The difference between Figs. 23 and 24 is shown in Fig. 25, in which the complement of pattern A with background noises due to the shift is shown. A thresholding process with index 160 (of 256) and three times the median filtering with a radius of one pixel are applied to Fig. 25 in order to filter the background noise. The result is shown in Fig. 26.

2) Red Light Filter: The effect of placing a red light filter (Hoya Red 25 A) in front of the camera lens to highlight the red light in the images is studied in this experiment. Digital images containing a traffic light are taken by a Kodak DVC323 USB digital color camera with a $6.2-\mathrm{mm}$ lens. The resolution of the 


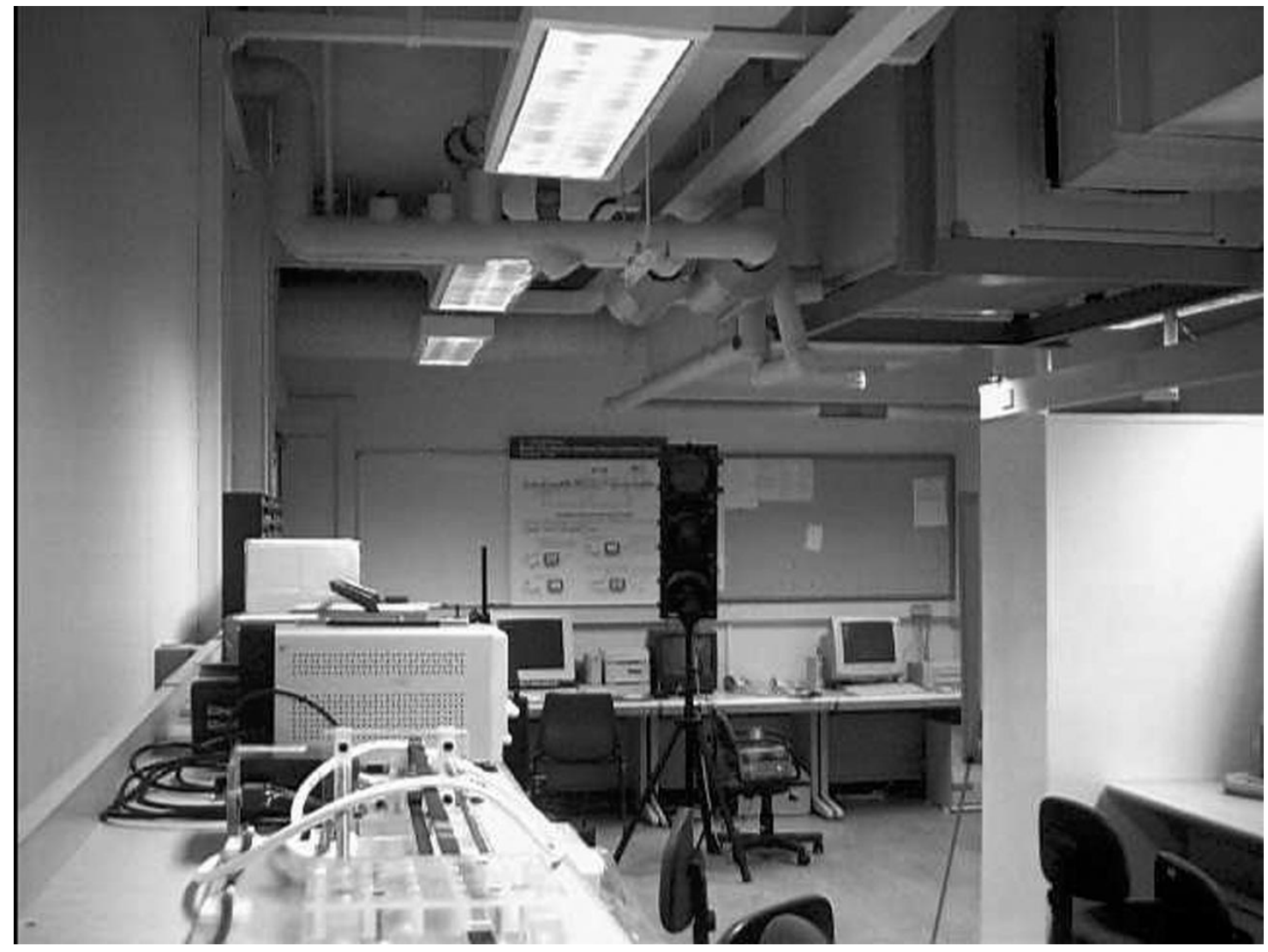

Fig. 28. Image with the LED traffic light turned off and shifted two pixels to the right.

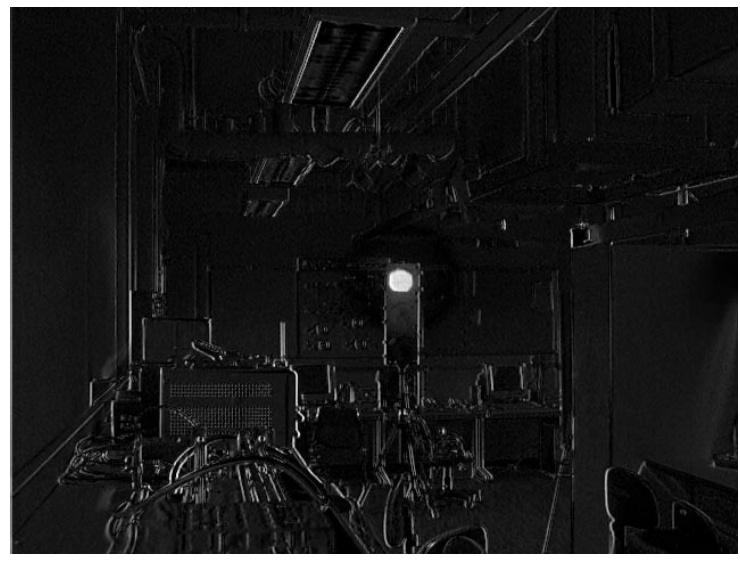

Fig. 29. The differenced image of Figs. 27 and 28 with noise.

image is $640 \times 480$ pixels. The distance between the LED light and the camera is about $8 \mathrm{~m}$. An image captured with the LED light turned on is shown in Fig. 27. Another image was captured with the LED turned off. The whole picture was then shifted to the right by two pixels, which is shown in Fig. 28. Figs. 27 and 28 are differenced, and the result is shown in Fig. 29.

After installing the red filter in front of the camera lens, an image was captured, which is shown in Fig. 30. Another one was captured with the LED light turned off, which was shifted to the right by two pixels (Fig. 31). Figs. 30 and 31 are differenced, and the result is shown in Fig. 32.

3) Discussions on Noise Reduction for Differencing: From the experimental results, the median filter is found to be effec- tive for removing small differencing noise. The mask of the median filter cannot be set to be too large. Otherwise, the LED signal may also be removed. The thresholding process is found to be useful for removing noise with smaller intensity than the LED signal in the differenced image. From the experimental results, much background noise is reduced.

The red light filter added before the camera lens is very effective in reducing the noise of differencing caused by the object and the camera movement. The filter can pass the red traffic light signal while reducing the intensity of the background. Thus, by differencing of the red images, the LED signal is readily seen. For an offset of two pixels without the red filter, the noise in the differenced image is high (Fig. 29). But for the same case with a red light filter (Fig. 32), the differenced noise is very low in size and intensity. Compared with the median filter and the thresholding method, the red filter method does not need extra processing time and gives better performance. For future research, a software filter can be used to select only the red signal instead of a physical red light filter. Moreover, intelligent methods for telling the camera to capture areas of interest (LED signal portion) can reduce much of the background noise.

\section{DISCUSSION AND CONCLUSIONS}

High-brightness LEDs are getting more popular and are opening up a number of new applications, especially with improved efficiency and new colors. In this paper, the novel idea based on the fast switching of LEDs is developed into a new kind of positioning beacon system. An LED traffic light or 


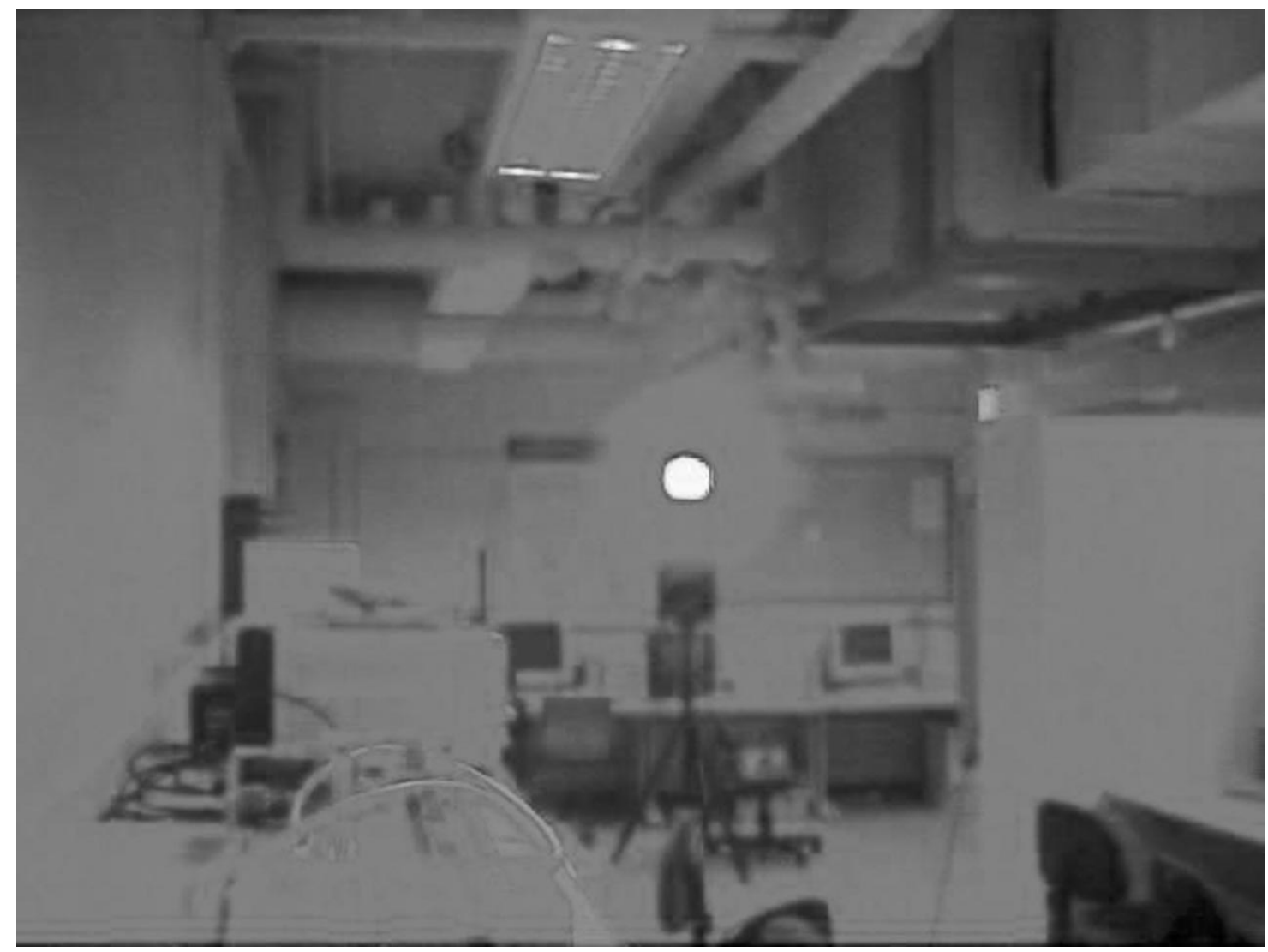

Fig. 30. LED turned on and with red filter in front of the camera lens.

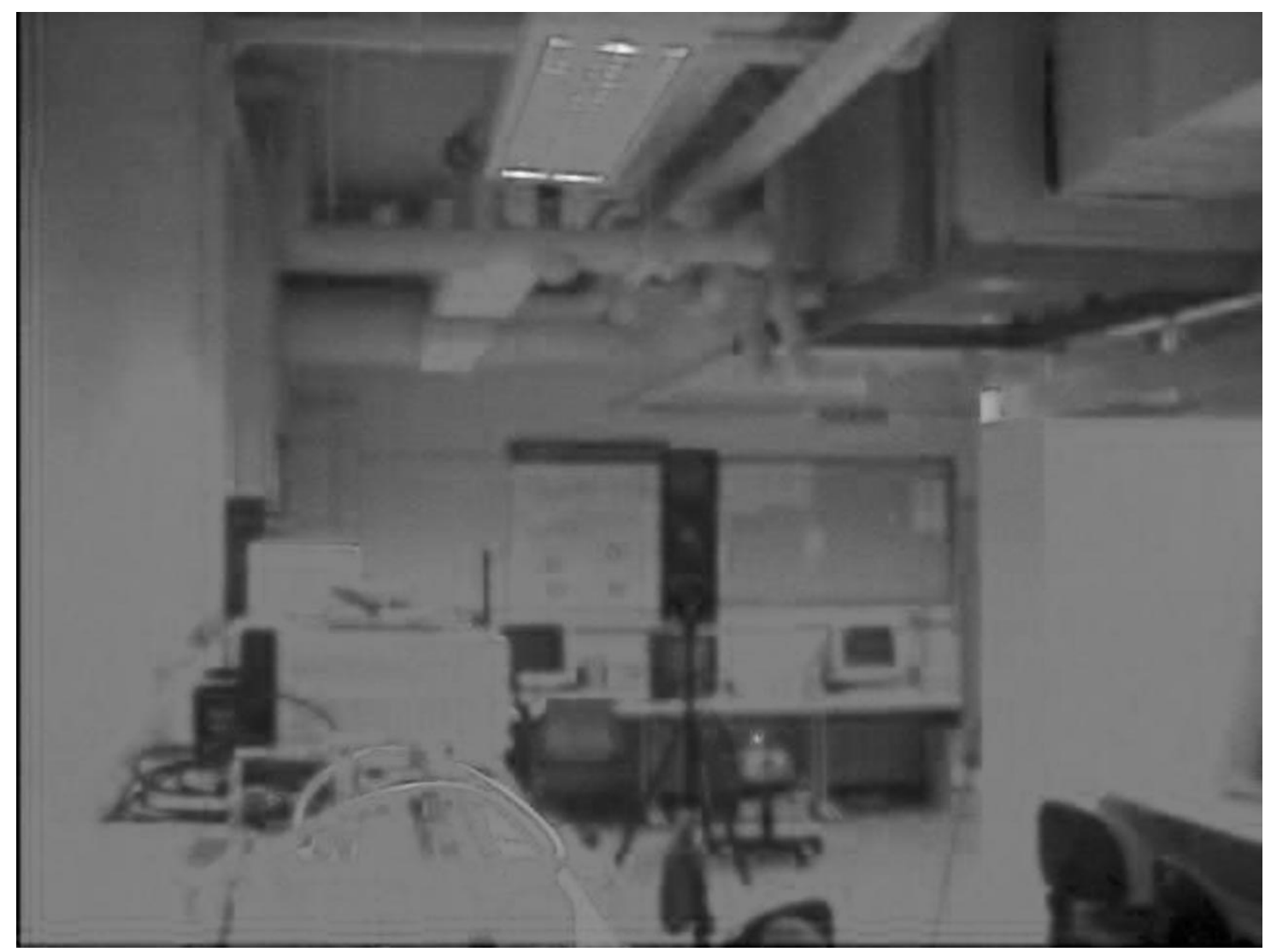

Fig. 31. LED traffic light turned off and with a red filter in front of the camera lens. This is shifted two pixels to the right relative to Fig. 30. 


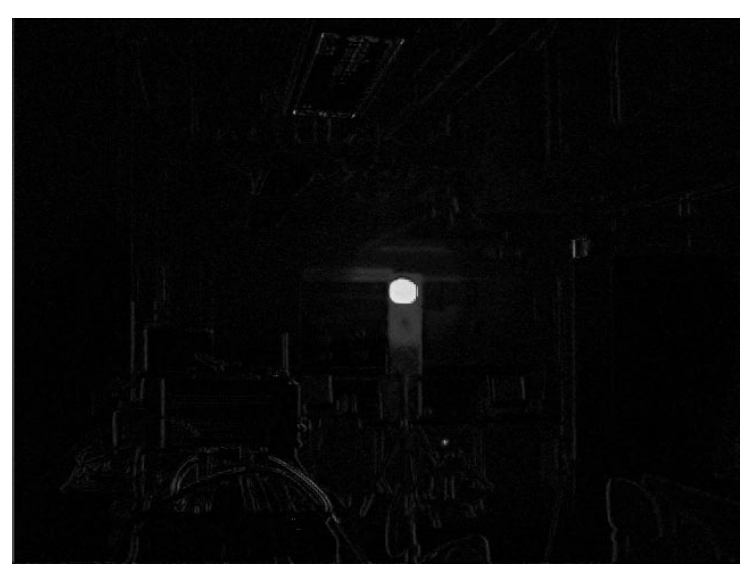

Fig. 32. The differenced image of Figs. 30 and 31 with much less noise.

signal device that can make use of visual light rays to transmit location code to a remotely located receiver is described. The system made up of high-brightness visible LEDs can provide the function of open-space wireless broadcasting of positioning information. It can also be used as an information beacon for short-distance communication.

As a medium for wireless short-range communication, visible light has both advantages and disadvantages when compared with infrared, microwave, and radio media. On one hand, LEDs are capable of high-speed operation and are available at low cost. Like the infrared, the visible spectral region is unregulated worldwide and FCC licenses are not necessary, as the commission does not regulate the visible light frequencies. Both infrared and visible light penetrate through glass, but not through walls. For the transmission to be possible, there must be no obstructions standing in the way of the visible LED light beam, as it requires a clear line of sight (LOS) between the sending side (LED) and the receiving side, whereas infrared also allows nondirected and non-LOS link design. With the use of a digital camera as a receiver reported in this paper, the view angle can be greatly improved. An interesting application given in this paper is an LED traffic light for the support of roadside-to-vehicle communications. There would be many other novel use of visible light from LEDs other than for broadcasting of positioning information. This concurrent use of LEDs for simultaneous signaling and communications will open up many new applications.

\section{REFERENCES}

[1] G. B. Stringfellow and M. G. Craford, "High brightness light emitting diodes," in Semiconductors and Semimetals. New York: Academic, 1997, vol. 48.

[2] M. G. Craford, "LED's challenge the incandescents," IEEE Circuits De vices, pp. 24-29, Sept. 1992.

[3] K. Werner, "Higher visibility for LED's," IEEE Spectrum, pp. 30-39, July 1994.

[4] D. Ibrahim and M. Beasley, "The benefits of LED traffic lights in London and the pilot test sites," in Inst. Elect. Eng.Conf. Road Transport Information and Control, Apr. 21-23, 1998, pp. 172-176.

[5] T. Saito, J. Shima, H. Kanemitsu, and Y. Tanaka, "Automobile navigation system using beacon information," Proc. IEEE Vehicle Navigation and Information System Conf. VNIS, pp. 139-145, 1989.

[6] S. Murata and T. Hirose, "Onboard locating system using real-time image processing for a self-navigating vehicle," IEEE Trans. Ind. Electron., vol. 40, pp. 145-154, Feb. 1993.
[7] G. Pang, E. Yang, C. Yeung, M. Hong, D. Yang, H. Liu, T. Kwan, L. Ko, and C. Chan, "Concurrent display and data communication using LED's,", Mar. 19, 1999.

[8] G. Pang, C. Chan, T. Kwan, and E. Yang, "Visible light communication for audio systems," IEEE Trans. Consumer Electron., vol. 45, pp. 1112-1118, Nov. 1999.

[9] G. Pang, H. Liu, C. Chan, and T. Kwan, "Vehicle location and navigation systems based on LED's," in Proc. 5th World Congr. Intelligent Transport Systems, Seoul, Korea, Oct. 12-16, 1998

[10] G. Pang, T. Kwan, C. Chan, and H. Liu, "LED traffic lights as a communication device," in Proc. Int. Conf. Intelligent Transportation Systems, Tokyo, Japan, Oct. 5-8, 1999, pp. 788-793.

[11] G. Pang, C. Chan, H. Liu, and T. Kwan, "Dual use of LEDs: Signaling and communications in ITS," in Proc. 5th World Congr. Intelligent Transport Systems, Seoul, Korea, Oct. 12-16, 1998.

[12] G. Pang, T. Kwan, H. Liu, and C. Chan, "Optical wireless based on high brightness visible LED's," Proc. IEEE Conf. Industry Applications, vol. 3, pp. 1693-1699, Oct. 1999.

[13] Y. Zhao, Vehicle Location and Navigation Systems. London, U.K.: Artech House, 1997.

[14] C. Drane and C. Rizos, Positioning Systems in Intelligent Transportation Systems. London, U.K.: Artech House, 1998.

[15] (1999) LCQ3: Speed Limits on Roads. Hong Kong Special Administrative Region Government. [Online]. Available: http://www.info.gov.hk/gia/general/199 901/13/0 113 203.htm

[16] J. O'Connell, The Philadelphia Story, U.K.: Traffic Technology International, 1997, pp. 106-110.

[17] (1997) OV5017. OmniVision Technologies, Inc.. [Online]. Available: http://www.ovt.com

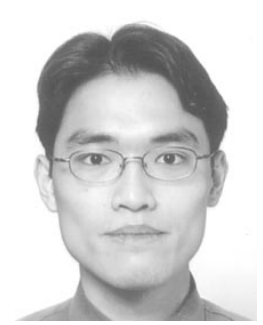

Hugh Sing Liu received the Bachelor degree and the M.Phil. degree from the Department of Electrical and Electronic Engineering, The University of Hong Kong, China, in 1997 and 2000, respectively.

His research thesis was titled "Integrated Vehicle Positioning System Using Sensors and Image Processing of Beacon Signal." He conducted various research works on vehicle location in the Industrial Automation Research Laboratory of The University of Hong Kong. His major activities include sensory circuits and systems, signal processing, and novel positioning methodologies development. His works resulted in more than ten conference and journal publications. In 2000, he was with the same Laboratory as a Design Engineer for an automatic inspection system project. He contributed to the design and development of an image acquisition and processing system using CMOS imaging sensors. Since 2001, he has been with Saning Electronic as an IC Design Engineer. He is responsible for a family of mixed-signal CMOS sensory integrated circuits products. His major works are on full custom, whole-chip circuit design and layout. His recent work includes research and development on electrostatic discharge protection on-chip circuitry.

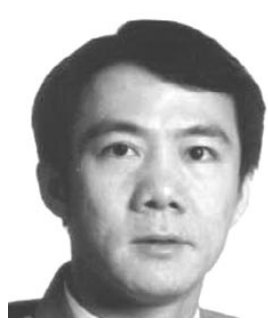

Grantham Pang (S'84-M'86-SM'01) received the Ph.D. degree from the University of Cambridge, U.K., in 1986.

He was with the Department of Electrical and Computer Engineering, University of Waterloo, Canada, from 1986 to 1996 and joined the Department of Electrical and Electronic Engineering at The University of Hong Kong in 1996. Since 1988, he has published more than 130 technical papers and has authored or coauthored eight books. His research interests include expert systems for control system design, intelligent control, intelligent transportation system, neural networks, control theory, and computer-aided design. He is an Editor of the International Journal of Intelligent Control and Systems, Control and Computers.

Dr. Pang is a member of the Institute of Electrical Engineers and of HKIE. He was the Organizing Chair of the 1996 IEEE Symposium on Computer-Aided Control System Design. He is a Chartered Electrical Engineer. 